\title{
EUROPEAN ORGANIZATION FOR NUCLEAR RESEARCH
}

15 September 1997

\section{Antiproton-proton annihilation at rest into $K_{L}^{\circ} K^{ \pm} \pi^{\mp} \pi^{\circ}-$ manifestations of isospin interference}

\begin{abstract}
A. Abele ${ }^{8)}$, J. Adomeit ${ }^{7)}$, C. Amsler ${ }^{15)}$, C.A. Baker ${ }^{5)}$, B.M. Barnett ${ }^{3)}$, C.J. Batty ${ }^{5)}$,
\end{abstract} M. Benayoun ${ }^{12)}$, A. Berdoz ${ }^{13)}$, K. Beuchert ${ }^{2)}$, S. Bischoff ${ }^{8)}$ P. Blüm ${ }^{8)}$, K. Braune ${ }^{11)}$, D.V. Bugg ${ }^{9)}$, T. Case $^{1)}$, O. Cramer ${ }^{11)}$, V. Credé $^{3)}$, K.M. Crowe ${ }^{1)}$, T. Degener ${ }^{2)}$, N. Djaoshvili ${ }^{8)}$, S. v. Dombrowski ${ }^{15, a)}$, M. Doser ${ }^{6)}$, W. Dünnweber ${ }^{11)}$, A. Ehmanns ${ }^{3)}$, D. Engelhardt ${ }^{8)}$, M.A. Faessler ${ }^{11)}$, P. Giarritta ${ }^{15)}$, R.P. Haddock ${ }^{10)}$, F.H. Heinsius ${ }^{1, b)}$, M. Heinzelmann ${ }^{15)}$, M. Herz ${ }^{3)}$, N.P. Hessey ${ }^{11)}$, P. Hidas ${ }^{4)}$, C. Hodd ${ }^{9)}$, C. Holtzhaußen ${ }^{8)}$ D. Jamnik ${ }^{11, \mathrm{c})}$, H. Kalinowsky ${ }^{3)}$, B. Kämmle ${ }^{7)}$, P. Kammel ${ }^{1)}$, J. Kisiel ${ }^{6, d)}$, E. Klempt ${ }^{3)}$, H. Koch ${ }^{2)}$, C. Kolo ${ }^{11, e)}$, M. Kunze ${ }^{2)}$, U. Kurilla ${ }^{2)}$, M. Lakata ${ }^{1)}$, R. Landua ${ }^{6)}$,

H. Matthäy ${ }^{2)}$, R. McCrady ${ }^{13)}$, J. Meier ${ }^{7)}$, C.A. Meyer ${ }^{13)}$, L. Montanet ${ }^{6)}$, R. Ouared $^{6)}$, F. Ould-Saada ${ }^{15)}$, K. Peters ${ }^{2)}$, B. Pick ${ }^{3)}$, C. Pietra ${ }^{15)}$, C.N. Pinder ${ }^{5)}$, M. Ratajcak ${ }^{2)}$, C. Regenfus ${ }^{11)}$, J. Reißmann ${ }^{7)}$, S. Resag ${ }^{3)}$, W. Roethel ${ }^{11)}$, P. Schmidt ${ }^{7)}$, I. Scott ${ }^{9)}$, R. Seibert ${ }^{7)}$, S. Spanier ${ }^{15)}$, H. Stöck ${ }^{2)}$, C. Straßburger ${ }^{3)}$, U. Strohbusch ${ }^{7)}$, M. Suffert ${ }^{14)}$, U. Thoma ${ }^{3)}$, M. Tischhäuser ${ }^{8)}$, C. Völcker ${ }^{11)}$, S. Wallis ${ }^{11)}$ D. Walther ${ }^{11, f)}$, U. Wiedner ${ }^{11)}$, B.S. Zou ${ }^{9)}$, and Č. Zupančičč ${ }^{11)}$

Crystal Barrel Collaboration

\begin{abstract}
The decay $\bar{p} p$ (at rest) $\rightarrow K_{L}^{\circ} K^{ \pm} \pi^{\mp} \pi^{\circ}$, studied on a sample of more than 14000 events, is found to proceed dominantly via two-body channels containing resonances with open strangeness. Contributions from $K_{1}(1270) \bar{K}$ and $K_{1}(1400) \bar{K}$, branching into the successive $K_{1}$-decay modes, and from $K^{*} \bar{K}^{*}, K^{*}(\bar{K} \pi)_{S}$ and $(K \pi)_{S}(\bar{K} \pi)_{S}$ are extracted by means of a partial-wave analysis which accounts well for the data and also allows a search for weak contributions from other $(K \pi \pi)$ and from $(K \bar{K} \pi)$ resonances. Striking asymmetries between the charged and the neutral kaonic resonances give evidence for interfering isospin 0 and 1 annihilation amplitudes.
\end{abstract}


Kaonic annihilation channels of protonium have been a profitable source of meson spectroscopy since the early bubble chamber work [1]. The $K \bar{K} \pi \pi$ channels give access to resonances in the open-strangeness $(K \pi \pi)$ and $(K \pi)$ and in the hidden-strangeness $(K \bar{K})$ and $(K \bar{K} \pi)$ systems.

A peculiarity of kaonic annihilation channels of protonium is the coherent contribution of the initial isospin $\mathrm{I}=0$ and 1 states. In contrast, protonium channels without kaons have fixed G-parity, $\mathrm{G}=\mathrm{C}(-1)^{I}$, which links the total isospin I to the incoherent $\mathrm{C}=+1$ and $\mathrm{C}=-1$ entrance channels so that $\mathrm{I}=0$ and 1 channels do not interfere. A $(K \bar{K})$-pair with net charge has isospin 1 and it can exist in a superposition of $\mathrm{G}=$ +1 spatially antisymmetric and $G=-1$ spatially symmetric states. The link between G-parity and spatial symmetry is a consequence of generalized Bose symmetry. Coupling of the charged $(K \bar{K})$-pair to pions yields protonium decay channels which are composed of $\mathrm{I}=0$ and 1 states with opposite G-parity and, hence, opposite kaon exchange symmetry $[1,2,3,4]$. The resulting isospin interference is therefore unambiguously evident via an intensity distribution asymmetric with respect to the exchange of the neutral and the charged kaon. So far, this effect has been studied in only one case, $\bar{p} p$ annihilation into $K_{S}^{\circ} K^{ \pm} \pi^{\mp}$, where the intermediate states $K^{* \pm} K^{\mp}$ and $(K \pi)_{S}^{ \pm} K^{\mp}$ are found to be slightly suppressed with respect to the corresponding neutral-neutral combinations [2].

We have studied $\bar{p} p$ annihilation at rest into $K_{L}^{\circ} K^{ \pm} \pi^{\mp} \pi^{\circ}$. The final sample of events is subjected to a partial-wave analysis in the framework of the isobar model. The rather complete description of the data, with only a small number of resonances, entails branching ratios and reveals the characteristics of isospin interference. In previous work on the equivalent $K_{S}^{\circ} K^{ \pm} \pi^{\mp} \pi^{\circ}$ channel $[4,5]$, statistics was poorer and no full partial-wave analysis was presented. However, evidence for a $\mathrm{I}=1 / 2, \mathrm{~J}^{P}=1^{+}$resonance, now termed $K_{1}(1270)$ [6], was obtained [5] and some charged/neutral asymmetries were noted qualitatively $[1,4,5]$.

Antiprotons from the Low Energy Antiproton Ring (LEAR) at CERN were stopped in a liquid hydrogen target in the center of the Crystal Barrel detector [7]. The incoming beam, with a $\bar{p}$ momentum of $200 \mathrm{MeV} / \mathrm{c}$ and an intensity of about $10^{4} \bar{p} / \mathrm{s}$, was defined

1) University of California, LBL, Berkeley, CA 94720, USA

2) Universität Bochum, D-44780 Bochum, Germany

3) Universität Bonn, D-53115 Bonn, Germany

4) Academy of Science, H-1525 Budapest, Hungary

5) Rutherford Appleton Laboratory, Chilton, Didcot OX110QX, UK

6) CERN, CH-1211 Genève 23, Switzerland

7) Universität Hamburg, D-22761 Hamburg, Germany

8) Universität Karlsruhe, D-76021 Karlsruhe, Germany

9) Queen Mary and Westfield College, London E14NS, UK

10) University of California, Los Angeles, CA 90024, USA

11) Universität München, D-80333 München, Germany

12) LPNHE Paris VI, VII, F-75252 Paris, France

13) Carnegie Mellon University, Pittsburgh, PA 15213, USA

14) Centre de Recherches Nucléaires, F-67037 Strasbourg, France

15) Universität Zürich, CH-8057 Zürich, Switzerland

a) Now at Cornell University, Cornell, Ithaka, NY, USA

b) Now at Universität Freiburg, Freiburg, Germany

c) University of Ljubljana, Ljubljana, Slovenia

d) University of Silesia, Katowice, Poland

e) This work is part of the Ph.D. thesis of C. Kolo

f) Now at Universität Bonn, Bonn, Germany 
by means of a silicon counter telescope in front of the target. The experiment made full use of the high momentum resolution and solid-angle coverage of the Crystal Barrel detector. Charged particles are detected by, successively, two proportional chambers with $\Omega=0.99 \times 4 \pi$ sr and a jet drift chamber with $\Omega=0.93 \times 4 \pi$ sr. Exposed to a magnetic field of $1.5 \mathrm{~T}$ parallel to the incident beam, the drift chamber measures momentum with a resolution that varies between $2 \%$ and $5 \%$ for transverse momenta between 0.1 and 0.5 $\mathrm{GeV} / \mathrm{c}$. The differential energy loss $\mathrm{dE} / \mathrm{dx}$, measured with the drift chamber, allows an identification of the charged kaons and pions which is sufficiently clear-cut for momenta below $400 \mathrm{MeV} / \mathrm{c}$. The two $\gamma$-rays from the $\pi^{\circ}$ decay are detected by a barrel-shaped electromagnetic calorimeter surrounding the drift chamber. It consists of $1380 \mathrm{CsI}(\mathrm{Tl})$ crystals, 16 radiation lengths deep, which are read out via wavelength shifters by photodiodes. The usable solid angle is $\Omega=0.95 \times 4 \pi \mathrm{sr}$, the angular resolution $\sigma \approx 20 \mathrm{mr}$ in both polar and azimuthal angles, and the energy resolution $\sigma / E \approx 2.5 \% / \sqrt[4]{E[G e V]}$. With a rather constant probability of about $50 \%$ for momenta above $200 \mathrm{MeV} / \mathrm{c}, K_{L}^{\circ}$ mesons escape the detector without interaction; in any case, they are identified by their missing mass.

The initial sample for event selection consists of 4.3 million minimum bias events and 4.5 million events recorded with a two-prong trigger corresponding to an enrichment factor of about 3.5 for the present channel. Of these, a total of 270000 events pass a preselection that requires two long tracks with hits in more than 9 layers of the drift chamber and with opposite curvature, an energy/momentum relation within generously defined kinematic boundaries and 2-4 separated energy deposits of at least $20 \mathrm{MeV}$ in the calorimeter that do not match with the charged-particle trajectories. Admitting one or two more energy deposits than required by $\pi^{\circ}(\rightarrow 2 \gamma)$ decay means that we accept events in which $K_{L}^{\circ}$ mesons interact in the calorimeter as well as events with "split-offs", i.e. secondaries produced by the charged mesons or photons. Such events were kept in the further selection and the surplus energy deposits were ignored if the energy deposits from $\pi^{\circ}$ decay could be identified without ambiguity. Separate treatment of the event samples corresponding to different numbers of unmatched energy deposits revealed no significant differences.

In the next step, cuts on differential energy loss $\mathrm{dE} / \mathrm{dx}$ versus momentum require one charged particle on the kaon branch and the other one on the pion branch, allowing for $1 \sigma$ deviation below the center of the kaon distribution and for $2 \sigma$ deviation above the center of the pion distribution, respectively. Taking the momentum correlation in the present channel into account, we estimate that $1 \%$ of these $\left(K^{ \pm} \pi^{\mp}\right)$ pairs have an ambiguous identification due to insufficient separation of the bands at high momenta. Applying in addition a gross $\pi^{\circ}$-cut on $\gamma \gamma$ invariant mass and a gross $K_{L^{\circ}}^{\circ}$ cut on the missing mass spectrum (which shows a clear peak at the $K_{L}^{\circ}$ mass), we are left with 21000 events.

Finally, a two-constraint kinematic fit is applied according to the hypothesis $\bar{p} p \rightarrow$ $K_{L}^{\circ}$ (missing) $K^{ \pm} \pi^{\mp} \pi^{\circ}$ with a $15 \%$ confidence level. Anticuts discard events which might be ascribed to the background channel $K_{S}^{\circ}\left(\rightarrow \pi^{+} \pi^{-}\right) K_{L}^{\circ} \pi^{\circ}$ and events with more than two unmatched energy deposits in the calorimeter out of which more than one credible $\pi^{\circ}$ could be reconstructed. The final sample consists of 14234 events.

Several background channels of the type $\pi^{+} \pi^{-} X, K^{ \pm} \pi^{\mp} X$ or $K^{+} K^{-} X$ (X symbolizing a set of neutral mesons) were studied by Monte Carlo simulations. Contributions to the final sample due to misidentified events from each one of these channels were found to 
be less than $1 \%$. However, the large number of potential background channels may give rise to an appreciable background with a rather flat distribution in phase space. In the data, the shape of the $(\mathrm{dE} / \mathrm{dx})$ distribution and the peak-to-background ratio in the missing mass spectrum (with nonfitted kinematic variables) permit two independent estimates of the total background, which consistently yield $10 \%$ of the final event number.

The total branching ratio is obtained from the subset of minimum bias data. Using the calculated final acceptance of $11 \%$ and taking background into account, we find $B R\left(\bar{p} p \rightarrow K_{L}^{\circ} K^{ \pm} \pi^{\mp} \pi^{\circ}\right)=(0.57 \pm 0.12) \%$ which is in agreement with the more accurate values from bubble chamber experiments $[4,5]$.

The final data set is presented in Figs. 1 and 2 in the form of invariant mass spectra for all possible combinations of two and three mesons, respectively. Since the decay probability of a four-body channel is a function of five independent kinematic variables, only parts of the full information contained in the data are preserved in these ten projections. All of them display strong deviations from mere phase-space distributions weighted by acceptance (shaded histograms).

The only prominent resonance visible in the four different $(K \pi)$ spectra of Fig. 1 is $K^{*}(892)$. No resonance structure is evident in the two spectra corresponding to a $(K \bar{K})+$ $(\pi \pi)$ division (lower panel of Fig. 1) where $a_{0}^{ \pm}(980)+\rho^{\mp}(770)$ is the only known primary resonance combination with the nominal mass inside our phase space.

A few indications of resonances are found in the three-meson invariant mass spectra (Fig. 2). In the case of $\left(K^{ \pm} \pi^{\mp} \pi^{\circ}\right)$, the most conspicuous structure shows up close to $1200 \mathrm{MeV}$. Its origin from $K_{1}(1270)$ becomes evident in the two-dimensional plots of this invariant mass versus $\mathrm{m}\left(K^{ \pm} \pi^{\circ}\right)$ and versus $\mathrm{m}\left(\pi^{ \pm} \pi^{\circ}\right)$ in Fig. 3, where its dominant decays, via $K^{* \pm}$ and $\rho^{ \pm}$, respectively, show up. In fact, the discovery of $K_{1}(1270)$ was the main outcome of a previous study of the present reaction [5]. Intensity maxima in Fig. 3 are shifted with respect to the PDG mass values due to the strong decrease of the available phase space near its boundaries. Additional broad structure in this representation of the data is attributed, in part, to reflections of different other reaction paths.

Another conspicuous structure in Fig. 2 consists of two narrow peaks at $1530 \mathrm{MeV} / \mathrm{c}^{2}$ and $1580 \mathrm{MeV} / \mathrm{c}^{2}$ in the $\left(K_{L}^{\circ} K^{ \pm} \pi^{\circ}\right)$ invariant mass spectrum. However, the upper position is consistent with that of monoenergetic background from channels with secondary $K^{+} \rightarrow \mu^{+} \nu$ decay of positive kaons stopped in the target (see [8]) where the $\mu^{+}$is easily misidentified as $\pi^{+}$. In a Monte-Carlo simulation, the corresponding background contribution from the present channel, which involves misidentification of a high-momentum $(\geq 400 \mathrm{MeV} / \mathrm{c}) \pi^{-}$as $K^{-}$, accounts for a substantial part of the peak at $1580 \mathrm{MeV} / \mathrm{c}^{2}$. So far, we have not found a convincing background source for the peak indicated at 1530 $\mathrm{MeV} / \mathrm{c}^{2}$, but we refrain here from speculations on its potential resonance origin.

Comparison of the invariant mass spectra of neutral and charged kaonic configurations in Figs. 1 and 2 reveals strong asymmetries. They may be qualitatively interpreted by a preference for a neutral-neutral combination in the primary reaction, if we assume that configurations with hidden strangeness play a minor role (as will be confirmed by the amplitude analysis below). The $K^{*}$ intensity pattern in the upper four panels of Fig. 1 indicates a predominance of the neutral-neutral combination for the $K^{*}+(\bar{K} \pi)$ and for the $\left(K^{*} \pi\right)+\bar{K}$ intermediate states. The former give rise to a $K^{*}$ signal only in the $K^{ \pm} \pi^{\mp}$ and $K_{L}^{\circ} \pi^{\circ}$ spectra, the latter only in the $K^{ \pm} \pi^{\mp}$ and $K^{ \pm} \pi^{\circ}$ spectra. The weakness of the corresponding charged-charged combinations which would show up only in $K_{L}^{\circ} \pi^{\mp}$ and $K^{ \pm} \pi^{\circ}$ or, respectively, only in $K_{L}^{\circ} \pi^{\mp}$ and $K_{L}^{\circ} \pi^{\circ}$, is evidenced by the relatively weak $K^{*}$ intensity in the $K_{L}^{\circ} \pi^{\mp}$ spectrum. The preference for neutral-neutral kaonic configura- 
tions in the primary reaction is also indicated in Fig. 2 by the more pronounced deviation from phase space in the $K_{1}(1270)$ region of the $\left(K^{ \pm} \pi^{\mp} \pi^{\circ}\right)$ spectrum in comparison to $\left(K_{L}^{\circ} \pi^{\mp} \pi^{\circ}\right)$.

As outlined at the beginning, the mere occurrence of asymmetry with respect to the exchange of $K^{ \pm}$and $K_{L}^{\circ}$ establishes unambiguously an interference of the two entrancechannel isospins, independent of assumptions of a primary two-body decay. The asymmetry with respect to the exchange of $\pi^{\mp}$ and $\pi^{\circ}$ (see middle panel of Fig. 1) implies a substantial contribution of spatially symmetric dipion states with isospin $i_{\pi \pi}=2$ instead of the naively expected complete dominance of the spatially antisymmetric dipion states with $i_{\pi \pi}=1$. Since the $i_{\pi \pi}=2$ interaction is repulsive it should not play a noticeable role in a process like $\bar{p} p$ annihilation which is governed by strong attractive final state interactions. In our channel, $i_{\pi \pi}=2$ dipion states are introduced mainly by the $(K \pi)+(\bar{K} \pi)$ intermediate states, with both unstable strange particles having isospin 1/2 like, e.g. $K^{*}+K^{*}$. In fact, the dipion states in the $(K \pi)+(\bar{K} \pi)$ configurations with total isospin $\mathrm{I}=1$ have uniquely $i_{\pi \pi}=2$ and contain no $i_{\pi \pi}=1$ component. The opposite is true for such configurations with $\mathrm{I}=0$ and, of course, for any strange intermediate resonance with isospin $1 / 2$ decaying into $K \pi \pi$, regardless of the initial isospin I. Therefore, coherent contributions of the entrance channel isospins are a possible, but not a necessary source of pionic asymmetries. Conversely we could, in principle, observe $K_{L}$ vs. $K^{ \pm}$asymmetry in the absence of $\pi^{\circ}$ vs. $\pi^{\mp}$ asymmetry.

The full 5-dimensional intensity distribution is subjected to a partial-wave analysis. It is assumed that the annihilation of protonium proceeds via a series of two-body decays into the final four-body channel. This is the customary "isobar-model" approach, justified mainly by its simplicity and its success in many other cases. Resonances are parametrized in the form of Breit-Wigner amplitudes, multiplied by L-dependent penetration factors [9] with range parameter $\mathrm{r}=1 \mathrm{fm}$. For the $(K \pi) \mathrm{S}$-wave, we use a parametrization [10] which fits the phase shifts extracted from the LASS data [11]. The dependence of the amplitudes on angle and spin variables is described by the helicity formalism. These amplitudes, for which an explicit expression is given in Ref. [12], are multiplied with complex coefficients $\alpha_{i}=a_{i} \cdot \exp \left(\mathrm{i} \phi_{i}\right)$ which are optimized by the fit. In addition, an incoherent background uniformly distributed in phase space is assumed to contribute to the total intensity, and is parametrized by a real positive fitting parameter. The fitting procedure uses a program [13] which incorporates the MINUIT [14] package and yields the unbinned maximum likelihood over the 5-dimensional phase space with acceptance weights derived from a Monte Carlo simulation of all detection and selection steps. Our partial wave analysis procedure is identical to that of Ref. [15] where it is described in more detail.

Results of these fits, which will be discussed in the following, are presented as full line histograms in Figs. 1 and 2 and in the form of rates listed in Tables 1 and 2. The relative rates of the two incoherent initial $\bar{p} p$ states and of the background are determined taking into account all interference effects of the exit channel resonances. The rates $r_{i}$ attributed to the individual resonances are obtained by integrating each of their intensities over the available phase space. The rates $r_{i}$ are normalized to add up to $100 \%$ for each initial state. They may be taken to be the relative rates of the resonances if interferences are neglected.

We restrict ourselves to the $\bar{p} p$ S-states $\left(J^{P C}=0^{-+}\right.$and $\left.1^{--}\right)$which are generally found to be dominant in annihilation at rest in liquid hydrogen [16]. Starting with a "minimal model" containing only a few resonances which are suggested by the gross features of the data to be present, we have stepwise included all contributions listed in Table 1. For contributions of several other resonances [6], allowed by the conservation 
laws, also from initial P-states, upper limits are given in Table 2.

By inclusion of the two $K_{1}(\rightarrow K \pi \pi)$ resonances (see Table 1 ) the fit quality is strongly improved which is expressed by a substantial increase of log likelihood (Fig. 4). The mass scans for a $K_{1}$ resonance with the PDG [6] width of $K_{1}(1270)$ and of $K_{1}(1400)$, in the respective left- and right-hand panels of Fig. 4, clearly demonstrate the presence of the neutral $\left(K^{ \pm} \pi^{\mp} \pi^{\circ}\right)$ versions of both resonances (full lines). In contrast, for the charged versions (dashed) the log likelihood does not peak at the respective resonance masses, giving evidence for a strong suppression of the charged $K_{1}$ contributions. The results listed in Table 1 were obtained with fixed $K_{1}$ masses and widths [6] but with secondary decay amplitudes taken as free fit parameters. We find reasonable agreement with the results obtained for the two $K_{1}$ resonances produced by $K^{ \pm}+p$ [17], except for the $(K \pi)_{S} \pi$ branch (see below).

In the $\left(K_{L}^{\circ} K^{ \pm} \pi^{\mp}\right)$ spectrum (Fig. 2) some weak excess intensity with respect to the fit is indicated slightly above $1400 \mathrm{MeV} / \mathrm{c}^{2}$. If the $h_{1}^{\prime}(1380)$ meson [18] is included in the fit, the spectrum is locally better reproduced and the result is consistent with our recent study of $\bar{p} p \rightarrow K_{L}^{\circ} K_{S}^{\circ} \pi^{\circ} \pi^{\circ}$ [15]. Part of the excess intensity may also be attributed to the $f_{1}(1420)$ meson which, however, could only be fed from protonium P-states (Table 2). With the present statistical uncertainty, no independent evidence can be established for these $(K \bar{K} \pi)$ resonances. No other contributions with hidden-strangeness resonances, including $a_{0}^{ \pm}(980)+\rho^{\mp}(770)$, are required by the fit (see Table 2$)$. For the sake of completeness, the two narrow peaks in the $K_{L}^{\circ} K^{ \pm} \pi^{\circ}$ spectrum, discussed above, are included as incoherent $\mathrm{X}^{ \pm} \pi$ contributions where $\mathrm{X}(1530)$ fits well when treated as a makeshift resonance with $J^{P}$ $=1^{+}, 2^{-}, 3^{+} \ldots$ and appropriate width and where $\mathrm{X}(1580)$ is parametrized in accordance with the proposed background mechanism. To assess the additional uncertainty due to different possible model spaces, we compared results for different "minimal-models" before our final fits and found that the intensities were stable within twice the given statistical errors and the phases within $\pm 20^{\circ}$, with the exception of contributions involving the $(K \pi)$ S-wave. The latter share a large ambiguity with the phase space distributed background, in particular when contributions from $\bar{p} p$ P-states are admitted (which easily absorb half of the quoted background). Hence, no quantitative conclusions should be drawn concerning contributions from initial P-states (assumed to be zero in Table 1) and from intermediate states involving the $(K \pi)$ S-wave.

The salient result of the partial wave analysis (Table 1, fit A) is a threefold selectivity, namely the strong predominance of

1. the $J^{P C}=1^{--}\left({ }^{3} S_{1}\right)$ initial state,

2. open-strangeness resonances in the exit channel,

3. neutral-neutral configurations in the exit channel.

Concerning point (1) note that the decay channels $(K \pi)_{S}(\bar{K} \pi)_{S}, K^{*}(\bar{K} \pi)_{S}$ and $K_{1} \bar{K}$, which constitute the bulk of the ${ }^{3} S_{1}$ decay, are forbidden by $J^{P}$ conservation for the ${ }^{1} S_{0}$ initial state.

As a rough measure of selectivity (3), the following charged/neutral rate ratios

$$
R_{c c / n n}=\left(\sum_{i} r_{i}^{c c}\right) /\left(\sum_{i} r_{i}^{n n}\right)
$$

are obtained from the results for fit A: $R_{c c / n n}\left({ }^{3} S_{1}\right)=0.33$ and $R_{c c / n n}\left({ }^{1} S_{0}\right)=0.9$. Separate summing over the decay branches of the dominant $K_{1} \bar{K}$ intermediate states yields $R_{c c / n n}\left({ }^{3} S_{1} \rightarrow K_{1}(1270) \bar{K}\right)=0.05$ and $R_{c c / n n}\left({ }^{3} S_{1} \rightarrow K_{1}(1400) \bar{K}\right)=0.35$. 
If all charged-charged combinations for the $1^{--}$initial state are excluded in the fit (Table 1, model B), the number of fit parameters is considerably reduced (from 46 to 26), while the log likelihood decreases by only 200 and the $\chi^{2} /$ bin averaged over all invariant mass spectra (Figures 1 and 2) increases from 1.47 to 1.52. This underlines the independence of the strong charged/neutral asymmetry on assumptions about the model space used for fitting.

The observed charge asymmetry provides evidence for interfering $I=0$ and $I=1$ annihilation amplitudes. For some of the annihilation paths like ${ }^{3} S_{1} \rightarrow K_{1}(1270) \bar{K}$, the interference causes almost complete suppression of the charged-charged meson combination. This implies almost complete isospin coherence, in accordance with theoretical expectations $[19,20,21,22,23]$, at least for the ${ }^{3} S_{1}$ state.

In addition, complete suppression would imply that the $I=0$ and 1 amplitudes have equal magnitude and a relative phase angle of either $0^{\circ}$ or $180^{\circ}$, depending on conventions in their definition; with our conventions [4, 24], this angle is $180^{\circ}$. As our results show, the two isospin amplitudes in the annihilation of the ${ }^{3} S_{1}$ state into $K_{L} K^{ \pm} \pi^{\mp} \pi^{\circ}$ are of comparable magnitude. In contrast, our result for the ${ }^{1} S_{0}$ state implies that its isovector amplitude for decay into $K_{L} K^{ \pm} \pi^{\mp} \pi^{\circ}$ is about twice as large in absolute magnitude as the isoscalar one and that their relative phase is within $10^{\circ}$ off $90^{\circ}$.

The situation in the $K_{S} K^{ \pm} \pi^{\mp}$ channel as studied by Conforto et al. [2] is different, though their spectra also clearly exhibit a stronger neutral $K^{*}$ as compared to its charged version. However, their analysis (see also Ref. [3]) yields $R_{c c / n n}\left({ }^{3} S_{1} \rightarrow K^{*}(892) \bar{K}\right)=0.7$ and $R_{c c / n n}\left({ }^{1} S_{0} \rightarrow K^{*}(892) \bar{K}\right)=0.4$. The isovector amplitude strongly dominates the annihilation from the ${ }^{3} S_{1}$ state, while for the ${ }^{1} S_{0}$ state the isoscalar amplitude is twice larger in absolute magnitude than the isovector one.

A study of more annihilation channels with open strangeness and different number of pions in the final state would be necessary to discern any systematics in the behaviour of the corresponding isospin amplitudes. Theoretical work, e.g. along the lines of Refs. $[19,20,21,22,23]$, might then throw more light on the mechanism of annihilation into channels with open strangeness and on the initial state interactions in the $\bar{N} N$ system, e.g. the role of the $\bar{n} n$ component of the protonium wave function.

\section{Acknowledgements}

We would like to thank the technical staff of the LEAR machine group and of all the participating institutions for their invaluable contributions to the success of the experiment. We acknowledge financial support the German Bundesministerium für Bildung, Wissenschaft, Forschung und Technologie, the Schweizerischer Nationalfonds, the British Particle Physics and Astronomy Research Council, the U.S. Department of Energy and the National Science Research Fund Committee of Hungary (contract No. DEFG03-87ER40323, DE-AC03-76SF00098, DE-FG02-87ER40315 and OTKA F014357). K. M. C., N. D. and F. H. H. acknowledge support from the Alexander von Humboldt Foundation.

\section{References}

[1] R. Armenteros and B. French in High Energy Physics, ed. by E.H.S. Burhop, Academic Press, London (1969) Vol. IV , p. 237.

[2] Conforto et al., Nucl. Phys. B3(1967)469 and Phys. Lett. 17(1965)170.

[3] A. Bettini et al., Nuovo Cim. 63A(1969)1199.

[4] N. Barash et al., Phys. Rev. B139(1965)1659. 
[5] A. Astier et al., Nucl. Phys. B10(1969)65.

[6] Review of Particle Physics: Phys. Rev. D54(1996)1.

[7] E. Aker et al., Nucl. Instr. Meth. A321(1992)69.

[8] A. Angelopoulos et al., Phys. Lett. B159(1985)210.

[9] F. von Hippel and C. Quigg, Phys. Rev. D5(1972)624.

[10] A. Abele et al., Phys. Lett. B (in press).

[11] D. Aston et al., Nucl. Phys. B296(1988)491.

[12] C. Amsler et al., Phys. Lett. B311(1993)362.

[13] C. Felix, program MAXTOOL (unpublished).

[14] CERN Program Library Entry D506, MINUIT (1992).

[15] A. Abele et al., accompanying letter.

[16] G. Reifenröther and E. Klempt, Phys. Lett. B245(1990)129.

[17] C. Daum et al., Nucl. Phys. B187(1981)1.

[18] D. Aston et al., Phys. Lett. B201(1988)573.

[19] I.L. Grach et al., Sov. J. Nucl. Phys. 48(1988)609.

[20] J. Carbonell, G. Ihle, J.M. Richard, Z. Phys. A334(1989)329.

[21] S. Furui, G. Strobel, A. Faessler, and R. Vinh Mau, Nucl. Phys. A516(1990)643.

[22] J. Jaenicke, B. Kerbikov, H.-J. Pirner, Z. Phys. A339(1991)297.

[23] C.B. Dover, J.-M. Richard, and J. Carbonell, Phys. Rev. C44(1991)1281.

[24] C. Kolo, Ph. D. thesis, Ludwig-Maximilians-Universität München, 1996. 


\begin{tabular}{|c|c|c|c|c|}
\hline \multicolumn{2}{|c|}{ a) Initial state ${ }^{3} S_{1}\left(J^{P C}=1^{--}\right)$, total rate: } & \multicolumn{3}{|c|}{$50 \%(\mathrm{~B})$} \\
\hline Amplitude & charge comb. & rate $(\mathrm{A})[\%]$ & rate $(\mathrm{B})[\%]$ & $\phi_{i}(B)\left[^{\circ}\right]$ \\
\hline \multirow[t]{2}{*}{$K^{*} \bar{K}^{*}(S=0)$} & $n n$ & 2.5 & 5.5 & 0 fixed \\
\hline & $c c$ & 1.0 & & \\
\hline \multirow[t]{2}{*}{$K^{*} \bar{K}^{*}(S=2)$} & $n n$ & 5.5 & 13.0 & 190 \\
\hline & $c c$ & 0.0 & & \\
\hline \multirow[t]{2}{*}{$(K \pi)_{S}(\bar{K} \pi)_{S}$} & $n n$ & 16.0 & 4.5 & 255 \\
\hline & $c c$ & 11.0 & & \\
\hline \multirow[t]{2}{*}{$\overline{K^{*}}(\overline{K \pi})_{S}$} & $n n$ & 3.5 & 4.0 & 240 \\
\hline & $c c$ & 1.0 & & \\
\hline \multirow[t]{2}{*}{$K_{1}(1270) \rightarrow K^{*} \pi L=0$} & $n n$ & 6.5 & 11.0 & 290 \\
\hline & $c c$ & 0.5 & & \\
\hline \multirow[t]{2}{*}{$K_{1}(1270) \rightarrow(K \pi)_{S} \pi L=1$} & $n n$ & 6.5 & 5.0 & 70 \\
\hline & $c c$ & 0.5 & & \\
\hline \multirow[t]{2}{*}{$K_{1}(1270) \rightarrow K \rho L=0$} & $n n$ & 5.0 & 11.0 & 80 \\
\hline & $c c$ & 0.0 & & \\
\hline \multirow[t]{2}{*}{$K_{1}(1400) \rightarrow K^{*} \pi L=0$} & $n n$ & 18.5 & 30.0 & 240 \\
\hline & $c c$ & 2.0 & & \\
\hline \multirow[t]{2}{*}{$K_{1}(1400) \rightarrow K^{*} \pi L=2$} & $n n$ & 2.5 & 8.0 & 25 \\
\hline & $c c$ & 1.0 & & \\
\hline \multirow[t]{2}{*}{$K_{1}(1400) \rightarrow(K \pi)_{S} \pi L=1$} & $n n$ & 9.0 & 8.0 & 20 \\
\hline & $c c$ & 7.5 & & \\
\hline \multicolumn{2}{|c|}{ b) Initial state ${ }^{1} S_{0}\left(J^{P C}=0^{-+}\right)$, total rate } & $13 \%(\mathrm{~A})$ & $11 \%(\mathrm{~B})$ & \\
\hline Amplitude & charge comb. & rate $(\mathrm{A})[\%]$ & rate $(\mathrm{B})[\%]$ & $\phi_{i}(B)\left[^{\circ}\right]$ \\
\hline \multirow[t]{2}{*}{$K^{*} \bar{K}^{*}(S=1)$} & $n n$ & 53.0 & 55.0 & 0 fixed \\
\hline & $c c$ & 47.0 & 45.0 & 310 \\
\hline \multicolumn{5}{|l|}{ c) Incoherent background: } \\
\hline \multicolumn{2}{|l|}{ Phase space distributed rate } & $14 \%(\mathrm{~A})$ & $31 \%(\mathrm{~B})$ & \\
\hline \multicolumn{2}{|l|}{$\mathrm{X}(1530)$ rate } & $2 \%(\mathrm{~A})$ & $3.5 \%(\mathrm{~B})$ & \\
\hline \multicolumn{2}{|l|}{$\mathrm{X}(1580)$ rate } & $2 \%(\mathrm{~A})$ & $4.5 \%(\mathrm{~B})$ & \\
\hline
\end{tabular}

Table 1: Results for the fit models A and B. All rates apply to the present $\left(K_{L}^{\circ} K^{ \pm} \pi^{\mp} \pi^{\circ}\right)$ final state and to $100 \%$ experimental acceptance. The standard errors, as obtained from MINUIT [14], amount to about $0.5 \%$ for rates up to $5 \%$, to about $(1 / 10)$ of the given values for larger rates, and to $\leq 10^{\circ}$ for $\phi$. 


\begin{tabular}{|c|c|c|c|c|c|}
\hline \multicolumn{4}{|c|}{ reaction channel } & total & $\operatorname{rate}(B)$ \\
\hline${ }^{3} S_{1}$ & $\longrightarrow$ & $K^{*}(1410) \bar{K}$ & $\left(K^{*} \pi, L=1\right) \bar{K}$ & $<$ & $1 \%$ \\
\hline${ }^{1} S_{0}$ & $\longrightarrow$ & $K^{*}(1410) \bar{K}$ & $\left(K^{*} \pi, L=1\right) \bar{K}$ & $<$ & $1 \%$ \\
\hline${ }^{3} S_{1}$ & $\longrightarrow$ & $K_{2}^{*}(1430) \bar{K}$ & $\left(K^{*} \pi, L=2\right) \bar{K}$ & $<$ & $2 \%$ \\
\hline${ }^{1} S_{0}$ & $\longrightarrow$ & $K_{2}^{*}(1430) \bar{K}$ & $\left(K^{*} \pi, L=2\right) \bar{K}$ & $<$ & $2.5 \%$ \\
\hline${ }^{3} S_{1}$ & $\longrightarrow$ & $a_{0}(980) \rho(770)$ & & $<$ & $1 \%$ \\
\hline${ }^{3} S_{1}$ & $\longrightarrow$ & $b_{1}(1280) \pi$ & $\left((K \pi)_{S} \bar{K}, L=0\right) \pi$ & $<$ & $1 \%$ \\
\hline${ }^{3} P_{0}$ & $\longrightarrow$ & $f_{1}(1420) \pi$ & $\left(K^{*} \bar{K}, L=0\right) \pi$ & $<$ & $2.5 \%$ \\
\hline${ }^{3} P_{1}$ & $\longrightarrow$ & $f_{1}(1420) \pi$ & $\left(K^{*} \bar{K}, L=0\right) \pi$ & $<$ & $2.5 \%$ \\
\hline${ }^{3} P_{2}$ & $\longrightarrow$ & $f_{1}(1420) \pi$ & $\left(K^{*} \bar{K}, L=0\right) \pi$ & $<$ & $2.5 \%$ \\
\hline${ }^{3} P_{0}$ & $\longrightarrow$ & $\eta(1440) \pi$ & $\left(K^{*} \bar{K}, L=1\right) \pi$ & $<$ & $1 \%$ \\
\hline
\end{tabular}

Table 2: Upper limits for other contributions relative to the total rate of the present final state, at confidence level 68\% (see Table 1). 

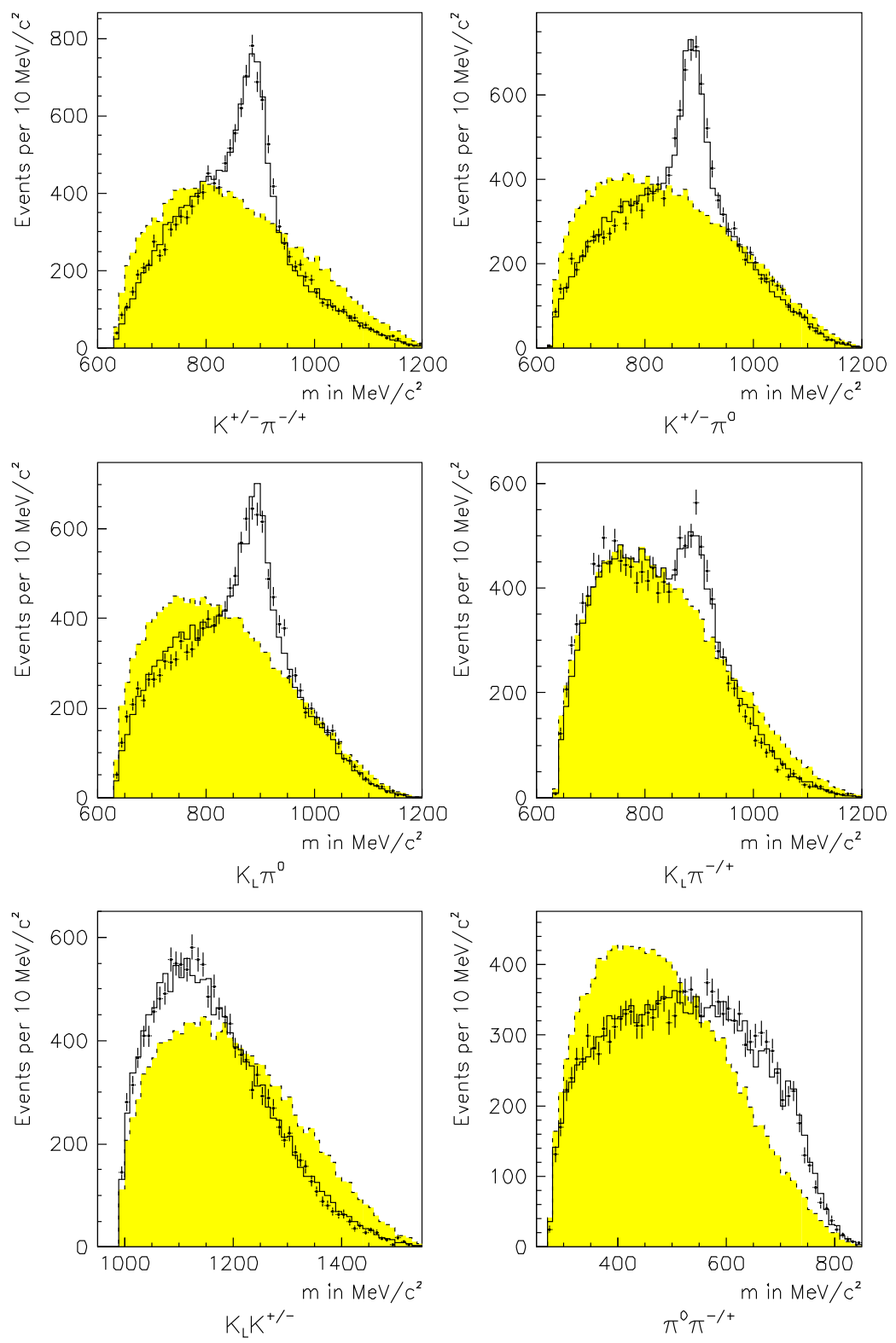

Figure 1: Invariant mass spectra for all two-meson combinations of the $\left(K_{L}^{\circ} K^{ \pm} \pi^{\mp} \pi^{\circ}\right)$ annihilation channel of protonium (full points with error bars representing one standard deviation). The full-line histogram shows the result of partial-wave fit B. The shaded histograms represent the phase space distribution obtained from a Monte-Carlo simulation. 

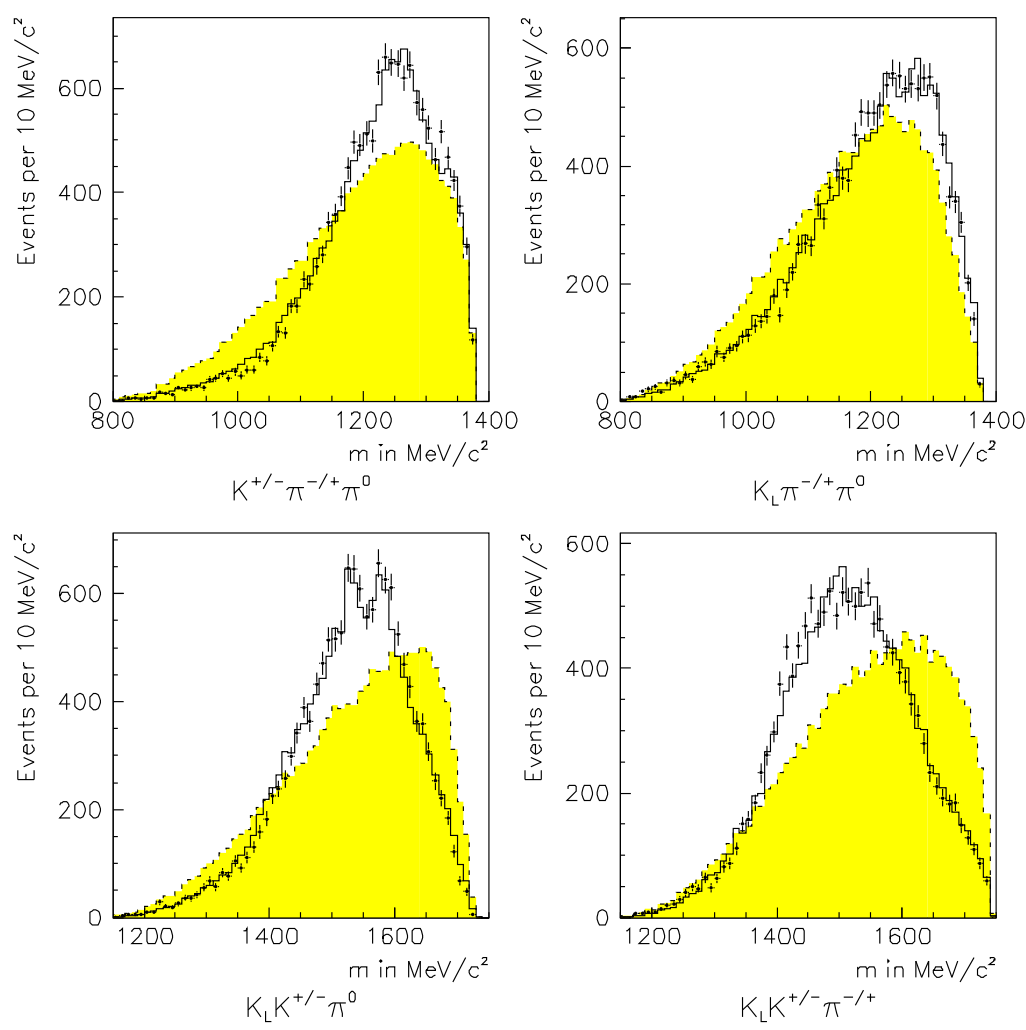

Figure 2: Same as Fig. 1, but for all three-meson combinations.
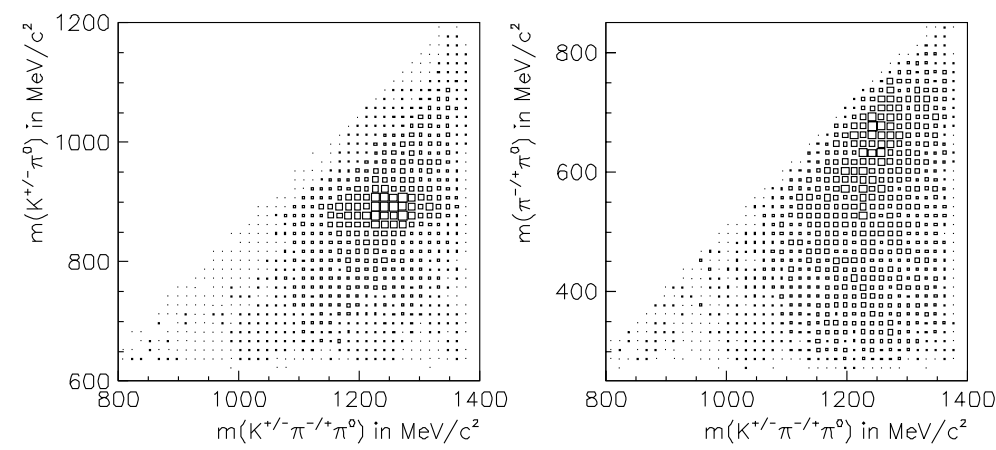

Figure 3: Two-dimensional projections of the data on $\left(K^{ \pm} \pi^{\mp} \pi^{\circ}\right)$ invariant mass versus $\left(K^{ \pm} \pi^{\circ}\right)$ and $\left(\pi^{\mp} \pi^{\circ}\right)$ invariant mass, respectively. 

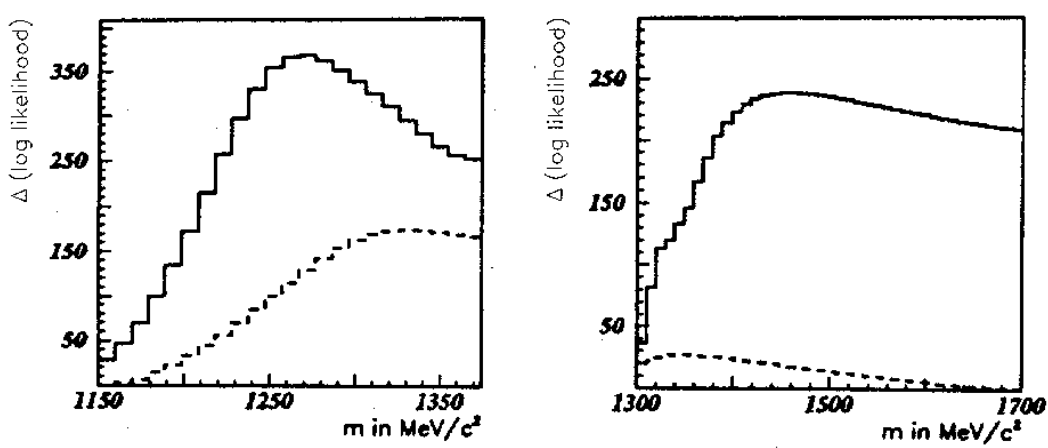

Figure 4: Log likelihood mass scan of an intermediate $K_{1}$ resonance in the neutral $\left(K^{ \pm} \pi^{\mp} \pi^{\circ}\right)$ and the charged $\left(K_{L} \pi^{\mp} \pi^{\circ}\right)$ system (full and dashed histograms, respectively). In addition to the $K_{1} \bar{K}$ channel, the fit model includes only $K^{*} \bar{K}^{*}, K^{*}(\bar{K} \pi)_{S}$ and $(K \pi)_{S}(\bar{K} \pi)_{S}$. In the left panel, the width of $K_{1}$ is fixed to the PDG [6] value for $K_{1}(1270)$, in the right panel to the $K_{1}(1400)$ value [6]. 